\title{
Proceedings
}

\section{On the Nature of the Substituent Effect in Slipped $\pi-\pi$ Structures ${ }^{\dagger}$}

\author{
Enrique M. Cabaleiro-Lago ${ }^{1, *}$, Jorge A. Carrazana-García ${ }^{1}$ and Jesús Rodriguez-Otero ${ }^{2}$ \\ 1 Departamento de Química Física, Facultade de Ciencias, Universidade de Santiago de Compostela, \\ Campus de Lugo, Avenida Alfonso X El Sabio s/n, 27002 Lugo, Spain \\ 2 Departamento de Química Física, Facultade de Química, Universidade de Santiago de Compostela, 15782 \\ Santiago de Compostela, Galicia, Spain \\ * Correspondence: caba.lago@usc.es \\ + Presented at the 24th International Electronic Conference on Synthetic Organic Chemistry, 15 November- \\ 15 December 2020; Available online: https://ecsoc-24.sciforum.net/.
}

Received: date; Accepted: date; Published: date

\begin{abstract}
In recent years, some controversy has been running related to the origin of the substituent effect in $\pi-\pi$ interactions. The earlier model based on ring polarisation and electrostatic interactions does not seem to be able to describe the observed effects, so a new model considering that substituent effect comes from the direct interaction between the substituent and the other ring has been proposed. In any case, the net effect of the substituent is largely dependent on other variables, and attention has been recently called upon the fact that the distance between rings can alter the observed behaviour. In a recent work (PCCP 2020, 22, 12068) we have shown that the origin of the substituent effect can change depending on the relative position of the substituent and the interacting rings. In the present study, an extension of the previous work is performed by using similar systems but changing the substituent, now located in a pyridine ring, and thus changing the relative positions of the substituent and the interacting rings.
\end{abstract}

Keywords: Intermolecular interactions; $\pi-\pi$ interactions; substituent effect

\section{Introduction}

The interactions involving aromatic rings can be modulated by incorporating substituents into the rings. However, the mechanism by which substituents introduce such modulation has been the subject of debate in recent years. In Hunter and Saunders model [1,2], the electrostatic interaction between rings is modified because the substituent adds or removes electronic density from the aromatic ring. However, theoretical results show that the interaction strengthens in benzene dimers unregards of the electron-donating or electron-withdrawing character of the substituent [3,4]. Wheeler and Houk proposed a new model that considers that the observed effect is due to direct through-space interactions between the substituent and the other molecule. Thus, it does not matter whether the substituent has electron-donor or electron withdrawing character $[5,6]$.

Riwar et al. have reported host-guest systems based on Rebek imide receptors designed in such a way that the relative position of two interacting rings can be controlled [7]. We have recently studied the nature of the substituent effect in these dimers, formed by di(isobutyramido)pyridine (1) and the Rebek imide receptors A and B (See Figure 1), showing that the relative position of the two interacting rings can change noticeably the nature of the substituent effect [8]. The substituent effect mostly follows changes on electrostatics mainly controlled by the direct interaction of the substituent and the other phenyl ring, whereas the contribution from the interacting rings is smaller. As the substituent is located further away the global result is a combination of both effects. 
In Riwar et al. systems, the substituent is located onto the phenyl ring of the Rebek receptor, interacting with the pyridine moiety of the di(isobutyramido)pyridine. In the present work, though, the substituent will be placed on the pyridine ring, thus changing the relative position between the substituent and the unsubstituted ring. Thus, in dimer 1-A the phenyl ring and the pyridine ring are stacked while the substituent is far from the phenyl ring, while in 1-B the rings are stacked but the substituent is located onto the unsubstituted ring, interacting directly with it (See Figure 1). This reverses the situation of the complexes 1-A and 1-B as compared to those in the previous work [8] (now the substituent is closer to the ring in 1-B). Similar computational tools are applied to get insight into the substituent in these new relative positions.

\section{Computational Details}

The isolated molecules 1, A, and B (See Figure 1) have been fully optimised at the PBE0-D3BJ def2-TZVP level (empirical dispersion correction included [9]) by using Gaussian09 [10], and the complexation energies have been obtained at the same level applying the counterpoise correction to avoid BSSE [11,12]. The analysis of the interaction will be carried out by using SAPT0/jun-cc-pVDZ [13]. SAPT allows the interaction energy to be obtained as a sum of contributions with physical meaning that can be identified with electrostatic, induction, exchange-repulsion and dispersion contributions [14-16]. The simplest SAPT level, SAPT0, is obtained by including the following terms:

$$
E_{\text {SAPTO }}=E_{\text {electr }}^{10}+E_{\text {exch }}^{10}+E_{\text {ind }}^{20}+E_{\text {exchind }}^{20}+E_{\text {disp }}^{20}+E_{\text {exchdisp }}^{20}+6 H F
$$

with $\delta H F$ being a term mostly representing induction to higher orders The contributions are usually

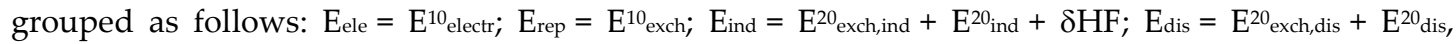
corresponding to electrostatic, repulsion, induction and dispersion contributions to the interaction energy.

The Functional Group Symmetry Adapted Perturbation Theory method (F-SAPT) as proposed by Parrish and Sherrill $[17,18]$ allows to split the total energy terms into contributions coming from user-defined fragments within the molecule. With such a partitioning, the effect of the substituent is estimated by comparing the results obtained for complexes containing substituted species with an unsubstituted dimer using an equivalent partition. All SAPT calculations have been performed with the PSI4 program [19]. Molecular structures are displayed with PyMOL [20].

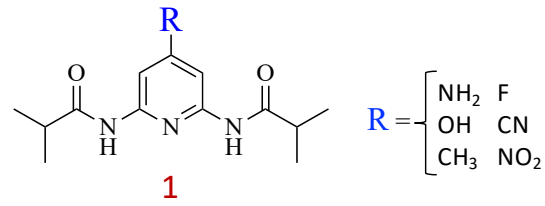

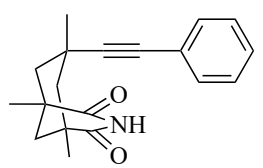

A

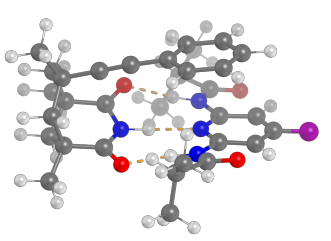

$1-\mathrm{A}$



B

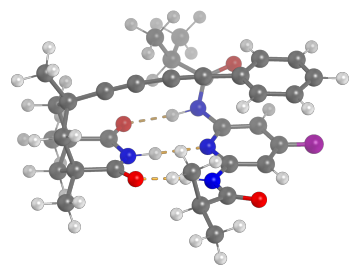

1-B

Figure 1. Systems considered in this work. Complexes with the fluorine derivative are presented to show the relative position of the substituent and the interacting rings. 


\section{Results}

Table 1 shows the values obtained after optimisation at the PBE0-D3BJ/def2-TZVP of the complexes 1-A and 1-B with the different substituents considered in the present work. It can be observed that all complexes show similar complexation energies, because the main interacting feature of the complex are the three hydrogen bonds established (Figure 1). 1-B complexes are around $2 \mathrm{kcal}$ $\mathrm{mol}^{-1}$ more stable than 1-A ones, and the most electron-withdrawing groups lead to the most stable complexes. The distances between rings are similar in all cases, though in 1-A complexes the phenyl ring centre is further from the substituent (negative values of $R_{\text {slip) }}$ than in $\mathbf{1 - B}$ complexes (positive values of Rslip).

Table 1. Optimised geometry $(\AA)$ and interaction energy $\left(\mathrm{kcal} \mathrm{mol}^{-1}\right)$ for dimers 1-A and 1-B at the PBE0-D3BJ/def2-TZVP level and F-SAPT0 energy contributions. Rcentr is the distance between ring centres, $R_{\text {vert }}$ is the distance between the centre of one ring and the plane containing the other, and Rslip is the slipped distance between ring centres.

\begin{tabular}{cccccccccccc}
\hline & \multicolumn{4}{c}{ PBE0-D3BJ/def2-TZVP } & \multicolumn{4}{c}{ F-SAPT0/jun-cc-pVDZ } \\
\hline $\mathbf{1 - A}$ & & $\Delta$ Eint & $\Delta \mathbf{E}_{\text {com }}$ & $\mathbf{R}_{\text {centr }}$ & $\mathbf{R}_{\text {ver }}$ & $\mathbf{R}_{\text {slip }}$ & Edis & Erep & Eele & Eind & Etot \\
\hline & $\mathbf{N H}_{2}$ & -27.83 & -24.20 & 3.728 & 3.368 & -1.599 & -26.72 & 48.74 & -39.49 & -13.41 & -30.88 \\
& $\mathbf{O H}$ & -28.08 & -24.57 & 3.684 & 3.356 & -1.519 & -26.58 & 48.08 & -39.46 & -13.17 & -31.14 \\
& $\mathbf{C H}$ & -27.84 & -24.35 & 3.695 & 3.361 & -1.536 & -26.91 & 47.78 & -38.76 & -12.95 & -30.83 \\
& $\mathbf{H}$ & -27.63 & -24.20 & 3.705 & 3.344 & -1.594 & -26.37 & 46.72 & -38.24 & -12.64 & -30.53 \\
& $\mathbf{F}$ & -28.17 & -24.83 & 3.686 & 3.362 & -1.512 & -26.30 & 47.12 & -39.13 & -12.85 & -31.15 \\
& $\mathbf{C N}$ & -28.72 & -25.58 & 3.627 & 3.352 & -1.385 & -26.94 & 46.74 & -39.03 & -12.61 & -31.83 \\
& $\mathbf{N O}_{2}$ & -28.81 & -25.69 & 3.616 & 3.352 & -1.357 & -27.07 & 46.90 & -39.20 & -12.66 & -32.04 \\
\hline $\mathbf{1 - B}$ & & $\Delta$ Eint & $\Delta E_{\text {com }}$ & $\mathbf{R}_{\text {centr }}$ & $\mathbf{R}_{\text {ver }}$ & $\mathbf{R}_{\text {slip }}$ & Edis & Erep & Eele & Eind & Etot \\
\hline & $\mathbf{N H}_{2}$ & -30.90 & -26.27 & 3.715 & 3.535 & 1.143 & -30.06 & 49.23 & -40.21 & -13.18 & -34.22 \\
& $\mathbf{O H}$ & -30.98 & -26.52 & 3.738 & 3.434 & 1.476 & -28.53 & 47.27 & -39.12 & -12.59 & -32.96 \\
& $\mathbf{C H}$ & -29.65 & -26.43 & 3.699 & 3.530 & 1.103 & -30.31 & 48.09 & -39.39 & -12.75 & -34.36 \\
& $\mathbf{H}$ & -30.24 & -25.84 & 3.697 & 3.532 & 1.091 & -29.07 & 46.84 & -38.76 & -12.52 & -33.51 \\
& $\mathbf{F}$ & -30.91 & -26.63 & 3.632 & 3.402 & 1.272 & -28.93 & 46.66 & -39.35 & -12.57 & -34.19 \\
& $\mathbf{C N}$ & -32.29 & -28.33 & 3.661 & 3.505 & 1.057 & -30.74 & 47.54 & -40.12 & -12.56 & -35.88 \\
& $\mathbf{N O}$ & -32.82 & -28.83 & 3.640 & 3.445 & 1.175 & -31.52 & 48.55 & -41.18 & -12.71 & -36.86 \\
\hline
\end{tabular}

The F-SAPT0/jun-cc-pVDZ decomposition in Table 1 indicates that the most stabilising contributions to the interaction energy are electrostatics and dispersion, while induction plays a minor though significant role. The great intensity of the three hydrogen bonds plus stacked rings is compensated by a large repulsion term reaching between 45 to $50 \mathrm{kcal} \mathrm{mol}^{-1}$. Focusing on the changes introduced by the substituent relative to the unsubstituted species, the trend shown in Figure 2 is obtained. It can be observed that changes are larger in 1-B dimers, because in these complexes the substituent lies closer to the phenyl ring, while in 1-A it is located further away (See Figure 1).

As in our previous work [8], the changes on the interaction energy in substituted species are mostly dominated by electrostatics. Both in structures 1-A and 1-B the electrostatic contribution changes significantly, especially with electron-withdrawing groups in structures 1-B. In 1-B dimers dispersion increases noticeably (mostly in the bulkier substituents) due to the closer proximity between the substituent and the phenyl ring. On the other hand, in 1-A dimers the changes associated to dispersion are smaller though still significant, because the relative position of the rings is quite similar but the substituent is not stacked onto the phenyl ring but located further away. Repulsion increases in most dimers (in 1-A dimers with electron-donor groups this effect is associated to an increase on repulsion between the pyridine ring and the carbonyl groups). Overall, the substituent effect is mainly controlled by electrostatics with contributions from dispersion when the substituent and the ring are close by. 

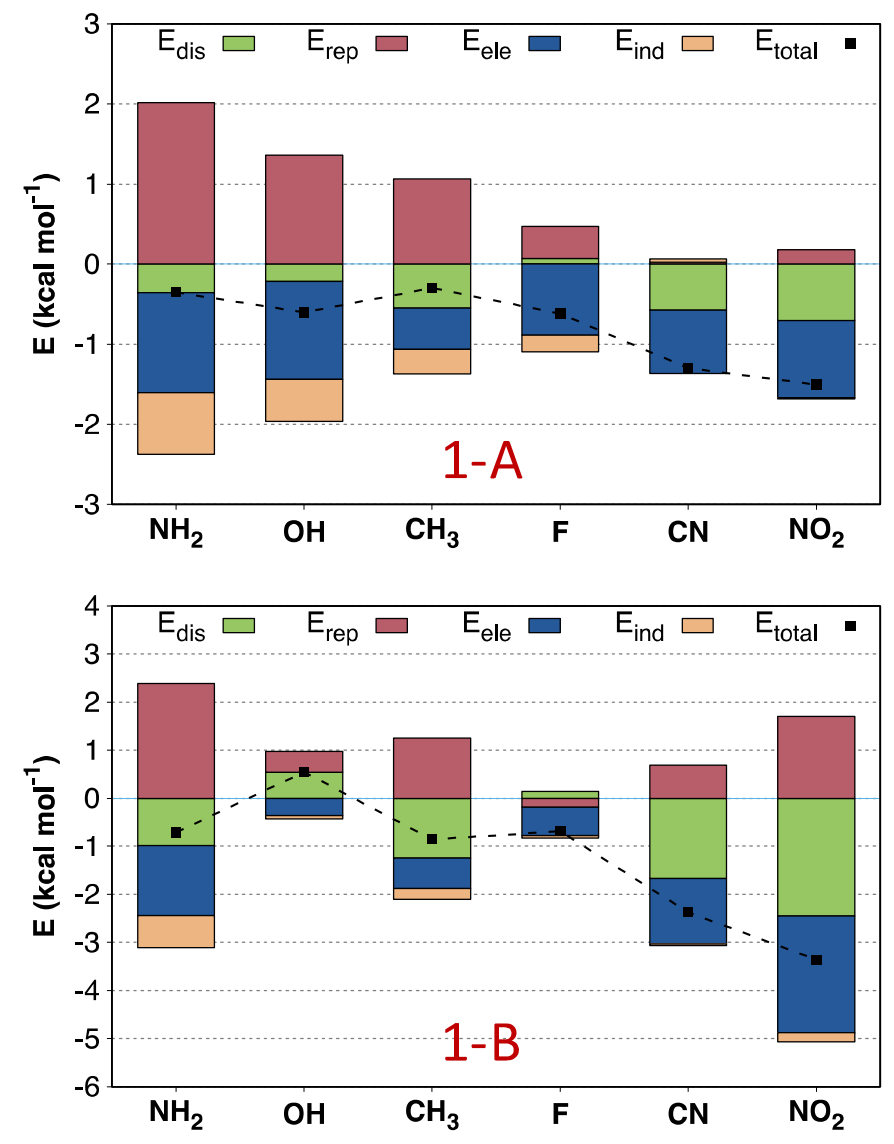

Figure 2. Energy differences in 1-A and 1-B relative to the unsubstituted species as obtained with the F-SAPT0/jun-cc-pVDZ.

Figure 3 shows the fragments defined for the species considered in this study as employed in the F-SAPT0 method, allowing to quantify the contribution from each of the fragment pairs in different molecules to the interaction energy components. Figure 4 depicts the total energy changes associated to each fragment. It can be observed that most of the changes are associated to the interaction of both rings and the substituent, while smaller contributions are observed coming from other regions of the molecules. Figure 5 shows the changes on the different contributions for complexes with $\mathrm{CN}$ and $\mathrm{CH}_{3}$. It can be observed that $\mathrm{CH}_{3}$ complexes show an increment in repulsion associated to the interaction between the pyridine ring and the NCO unit. This change is larger in 1A complex, while in 1-B there is also a repulsive contribution coming from the interaction of the methyl group with the ring. There are also slight favourable changes in induction and electrostatics (between pyridine and NCO) and dispersion (between rings). In 1-B complex the behaviour is similar, but with a large contribution from dispersion due to the direct contact between the methyl group and the ring. In the case of the $\mathrm{CN}$ derivatives the behaviour is clearly different. The repulsion between the pyridine ring and NCO group decreases. There are larger electrostatics, dispersion and repulsion contributions associated to the phenyl ring. In 1-B complexes, the same behaviour is observed though more markedly. There are large stabilising changes in electrostatics and dispersion associated to the interaction of the substituent with the phenyl ring, while repulsion decreases,

These trends can be observed in Figure 6, showing the energy changes associated to the interaction of the fragments defined for species $\mathbf{A}$ and $\mathbf{B}$ (Figure 3) and the fragments in $\mathbf{1}$ (pyridine ring, substituent and both isopropylamido groups). It can be observed that the substituent mostly affects to the interaction with the phenyl group in $\mathbf{A}$ and $\mathbf{B}$, while the interaction with other groups as the ethinyl linkers, or the NCO unit exhibit much smaller variations. In 1-A dimers, the changes on the interaction between the substituent and the phenyl group are smaller than in 1-B ones, and 
roughly follow the trend on electron-withdrawing capacity, being especially large for $\mathrm{CN}$ and $\mathrm{NO}_{2}$ substituents. The changes associated to the interactions with the pyridine ring are much smaller, in some cases dominated by the interaction with the phenyl ring, but with similar contributions from the ethinyl linkers and NCO groups. The changes associated to the isopropylamido groups are much smaller except in the case of the $\mathrm{OH}$ derivative.
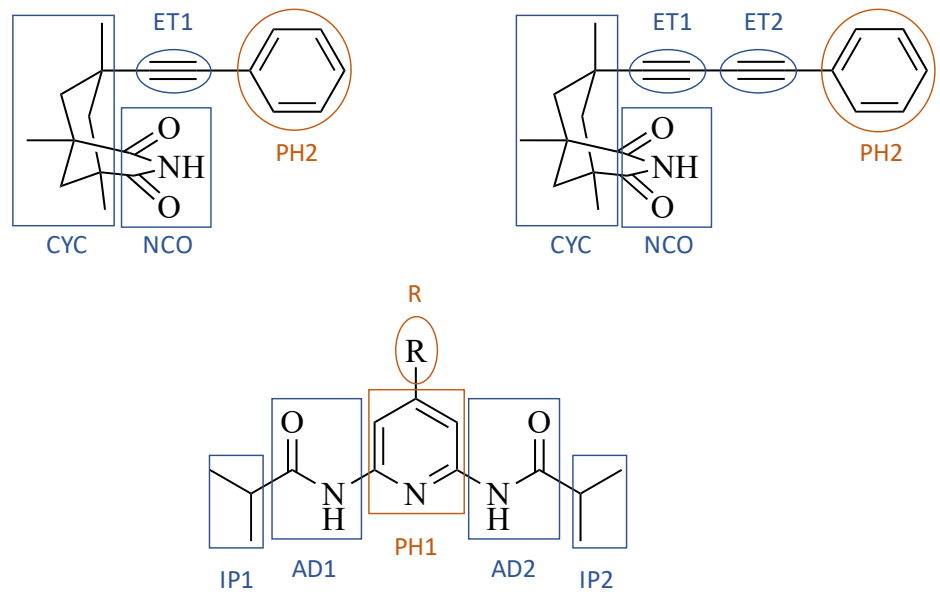

Figure 3. Schematic representation of the species forming the dimers 1-A and 1-B, showing the groups on which each of the molecules is decomposed in the F-SAPT0 calculations.

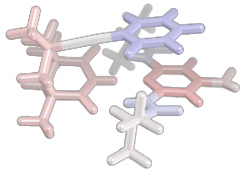

$\mathrm{NH}_{2}$

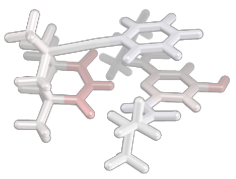

$\mathrm{OH}$



$\mathrm{CH}_{3}$

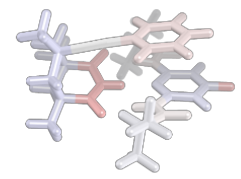

$\mathbf{F}$

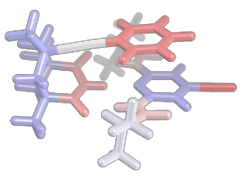

CN

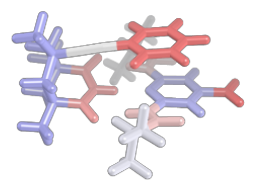

$\mathrm{NO}_{2}$ 1-A



$\mathrm{NH}_{2}$

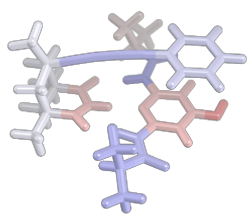

$\mathrm{OH}$

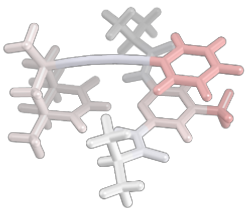

$\mathrm{CH}_{3}$

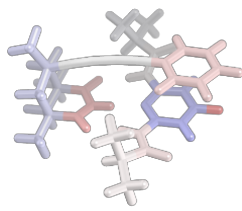

F



CN

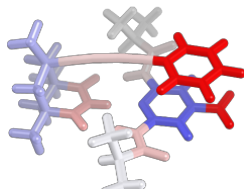

$\mathrm{NO}_{2}$

Figure 4. Contributions (relative to unsubstituted complexes) to the total interaction energy in dimers 1-A and 1-B considering the partitioning shown in Figure 3 using the F-SAPT0 method, coloured by the global contributions of each group. The colour scale goes from $-3 \mathrm{kcal} \mathrm{mol}^{-1}$ (red) to $+3 \mathrm{kcal} \mathrm{mol}^{-1}$ (blue). 




1-A

$E_{\text {ele }}$

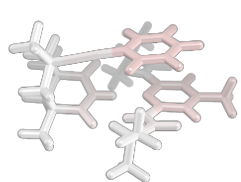

$E_{\text {dis }}$

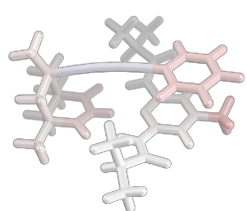

1-B

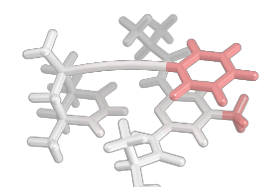

$E_{\text {dis }}$

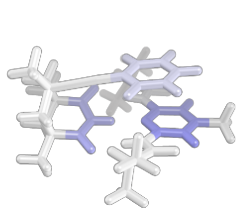

$E_{\text {rep }}$

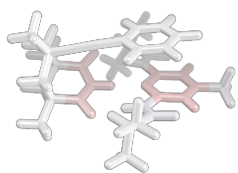

$E_{\text {ind }}$



$E_{\text {rep }}$

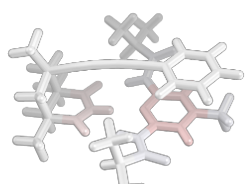

$E_{\text {ind }}$

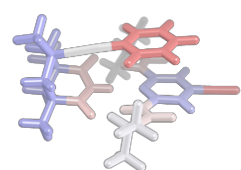

$E_{\text {ele }}$

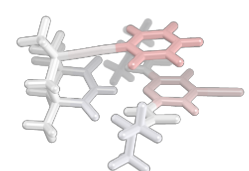

$E_{\text {dis }}$



$E_{\text {ele }}$



$E_{\text {dis }}$

$\mathrm{CN}$

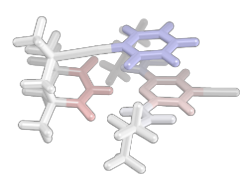

$E_{\text {rep }}$

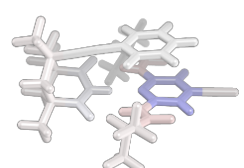

$E_{\text {ind }}$

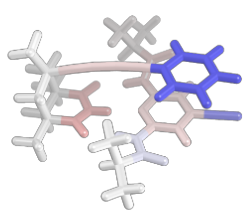

$E_{\text {rep }}$

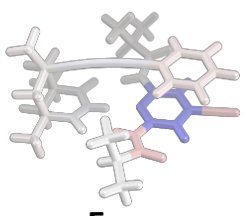

$E_{\text {ind }}$

\section{$\mathrm{CH}_{3}$}

Figure 5. Contributions (relative to unsubstituted complexes) in dimers 1-A and 1-B considering the partitioning shown in Figure 3 using the F-SAPT0 method, coloured by the global contributions of each group. The colour scale goes from $-3 \mathrm{kcal} \mathrm{mol}^{-1}$ (red) to $+3 \mathrm{kcal} \mathrm{mol}^{-1}$ (blue). 

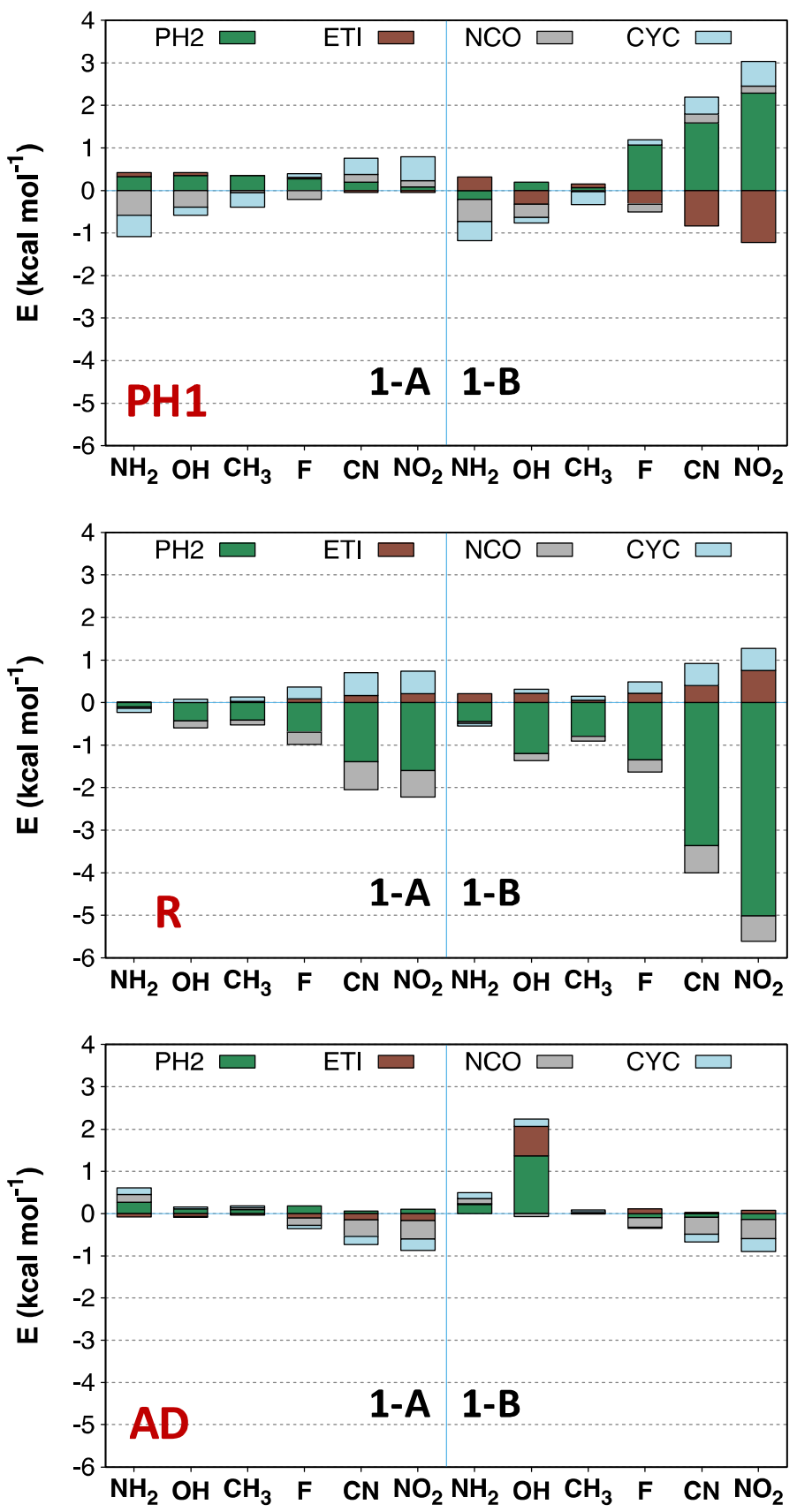

Figure 6. Contributions to the interaction energy changes relative to the unsubstituted species that originate in interactions between the pyridine ring PH1 (top), the substituent (middle) and the amide groups (bottom) in $\mathbf{1}$ with the different fragments defined in species A and B. F-SAPT0/jun-cc-pVDZ.

Figure 7 shows the contributions of the different terms of the interaction energy coming from the interaction between rings and between the phenyl ring and the substituent. Considering the changes associated to the substituent it becomes clear that they are dominated by electrostatics, though in 1-B structures dispersion contributes significantly. The changes associated to the polarization of the ring are much smaller, and almost negligible in 1-A dimers, though the effect is similar to that of the substituent in electron-donor groups. In 1-B dimers, the changes associated to the ring are larger but clearly overcome by the contributions from the substituent. Thus, as in our previous work, the substituent effect is mostly controlled by the direct interaction of the substituent 
and the ring (mainly from electrostatics), though when the distance between ring and substituent increases, the contributions from the polarised ring can also be of significance.
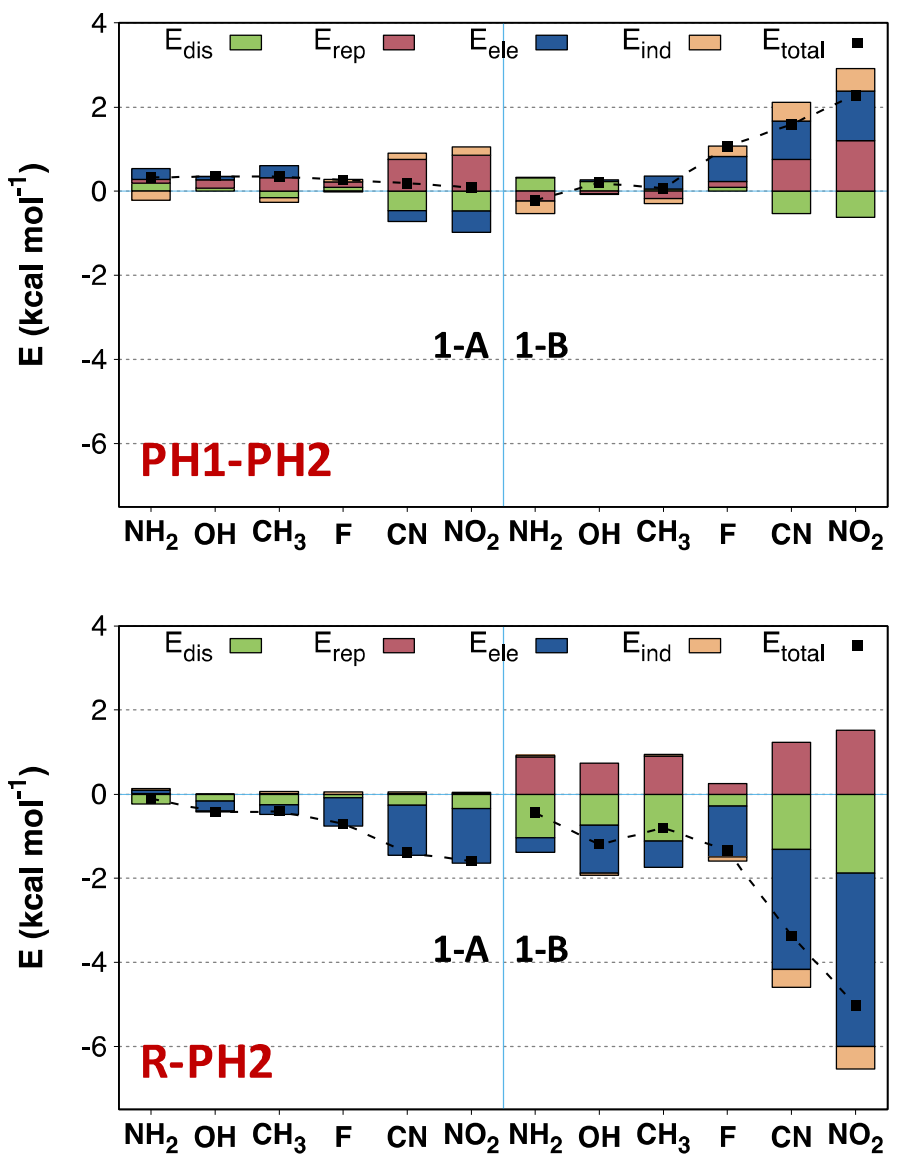

Figure 7. Contributions to the interaction energy changes relative to the unsubstituted species that originate in interactions between the pyridine ring PH1 (top) and R (bottom) in $\mathbf{1}$ with the phenyl ring PH2 in molecules A and B. F-SAPT0/jun-cc-pVDZ.

\section{Conclusions}

The influence of the relative positions of the rings upon the substituent effect in $\pi-\pi$ interactions has been computationally studied. The changes observed in the stability of the complexes have been analysed using F-SAPT0, providing not only information about the nature of the interaction and its controlling factors, but also allowing to quantify the contributions coming from different regions of the interacting species.

The substituent effect is mostly controlled by the contribution coming from the direct interaction of the substituent and the phenyl ring, mainly coming from changes on electrostatics and dispersion. The changes associated to the interaction between pyridine and phenyl rings are smaller and usually oppose the contribution from the substituent. Only when the substituent is located further away from the phenyl ring its contribution decreases enough as to make the contribution coming from the interacting rings to be of significance.

\section{References}

1. Hunter, C.A.; Sanders, J.K.M. The nature of .pi.-.pi. interactions. J. Am. Chem. Soc. 1990, 112, 5525-5534, doi:10.1021/ja00170a016. 
2. Hunter, C.A.; Lawson, K.R.; Perkins, J.; Urch, C.J. Aromatic interactions. J. Chem. Soc. Perkin Trans. 2001, 2, 651-669, doi:10.1039/B008495F.

3. Sinnokrot, M.O.; Sherrill, C.D. Substituent Effects in $\pi-\pi$ Interactions: Sandwich and T-Shaped Configurations. J. Am. Chem. Soc. 2004, 126, 7690-7697, doi:10.1021/ja049434a.

4. Sinnokrot, M.O.; Sherrill, C.D. Unexpected Substituent Effects in Face-to-Face $\pi$-Stacking Interactions. J. Phys. Chem. A 2003, 107, 8377-8379, doi:10.1021/jp030880e.

5. Wheeler, S.E.Understanding substituent effects in noncovalent interactions involving aromatic rings. Acc. Chem. Res. 2013, 46, 1029-1038, doi:10.1021/ar300109n.

6. Wheeler, S.E.; Houk, K.N.Origin of substituent effects in edge-to-face aryl-aryl interactions. Mol. Phys. 2009, 107, 749-760, doi:10.1080/00268970802537614.

7. Riwar, L.-J.; Trapp, N.; Kuhn, B.; Diederich, F. Substituent Effects in Parallel-Displaced $\pi-\pi$ Stacking Interactions: Distance Matters. Angew. Chem. Int. Ed. 2017, 56, 11252-11257, doi:10.1002/anie.201703744.

8. Cabaleiro-Lago, E.M.; Rodríguez-Otero, J.; Vázquez, S.A.The relative position of $\pi-\pi$ interacting rings notably changes the nature of the substituent effect. Phys. Chem. Chem. Phys. 2020, 22, 12068-12081, doi:10.1039/D0CP01253J.

9. Grimme, S.Density functional theory with London dispersion corrections. WIREs Comput. Mol. Sci. 2011, 1, 211-228, doi:10.1002/wcms.30.

10. Frisch, M.J.; Trucks, G.W.; Schlegel, H.B.; Scuseria, G.E.; Robb, M.A.; Cheeseman, J.R.; Scalmani, G.; Barone, V.; Mennucci, B.; Petersson, G.A.; et al. Gaussian 09, revision A.02. 2009.

11. Boys, S.F.; Bernardi, F.The calculation of small molecular interactions by the differences of separate total energies. Some procedures with reduced errors. Mol. Phys. 1970, 19, 553-566, doi:10.1080/00268977000101561.

12. Chalasinski, G.; Szczesniak, M.M. State of the Art and Challenges of the ab Initio Theory of Intermolecular Interactions. Chem. Rev. 2000, 100, 4227-4252.

13. Parker, T.M.; Burns, L.A.; Parrish, R.M.; Ryno, A.G.; Sherrill, C.D. Levels of symmetry adapted perturbation theory (SAPT) I. Efficiency and performance for interaction energies. J. Chem. Phys. 2014, 140, 094106, doi:10.1063/1.4867135.

14. Jansen, G. Symmetry-adapted perturbation theory based on density functional theory for noncovalent interactions. WIREs Comput. Mol. Sci. 2014, 4, 127-144, doi:10.1002/wcms.1164.

15. Szalewicz, K. Symmetry-adapted perturbation theory of intermolecular forces. WIREs Comput. Mol. Sci. 2012, 2, 254-272, doi:10.1002/wcms.86.

16. Patkowski, K. Recent developments in symmetry-adapted perturbation theory. WIREs Computational Mol. Sci. 2020, 10, e1452, doi:10.1002/wcms.1452.

17. Parrish, R.M.; Parker, T.M.; David Sherrill, C. Chemical assignment of symmetry-adapted perturbation theory interaction energy components: The functional-group SAPT partition. J. Chem. Theory Comput. 2014, 10, 4417-4431, doi:10.1021/ct500724p.

18. Parrish, R.M.; Sherrill, C.D. Spatial assignment of symmetry adapted perturbation theory interaction energy components: The atomic SAPT partition. J. Chem. Phys. 2014, 141, 044115, doi:10.1063/1.4889855.

19. Parrish, R.M.; Burns, L.A.; Smith, D.G.A.; Simmonett, A.C.; DePrince, A.E.; Hohenstein, E.G.; Bozkaya, U.; Sokolov, A.Y.; Di Remigio, R.; Richard, R.M.; et al. Psi4 1.1: An Open-Source Electronic Structure Program Emphasizing Automation, Advanced Libraries, and Interoperability. J. Chem. Theory Comput. 2017, 13, 3185-3197, doi:10.1021/acs.jctc.7b00174.

20. Schrödinger, L.L.C. The PyMOL Molecular Graphics System; Version 2.1; Open-Source, 2.1; 2015.

Publisher's Note: MDPI stays neutral with regard to jurisdictional claims in published maps and institutional affiliations.



(C) 2020 by the authors. Submitted for possible open access publication under the terms and conditions of the Creative Commons Attribution (CC BY) license (http://creativecommons.org/licenses/by/4.0/). 